\title{
Efectos de la infusión aguda de octreotido sobre la función renal en pacientes con cirrosis hepática e hipertensión portal
}

\author{
G uillermo Silva $P^{1}$, Roberto Segovia $M^{1,2}$, \\ Claudia Backhouse $\mathrm{E}^{3 a}$, Mariana Palma $2 \mathrm{a}$, \\ Sylvia Márquez², Hernán Iturriaga $R^{2,3}$. \\ Effects of acute octreotide infusion on \\ renal function in patients with cirrhosis \\ and portal hypertension
}

Background: Octreotide is used in the treatment of acute variceal bleeding, based on its inhibitory effects of post-prandial splanchnic hyperemia and splanchnic venoconstriction. The consequences of these haemodynamic changes on renal circulation are not well known in cirrhotic patients. Aim: To evaluate the effects of acute octreotide administration on several parameters of renal function, including free water clearance, in patients with cirrhosis with or without ascites. Patients and Methods: Twenty cirrhotic patients, Child-Pugh A or B, with or without ascites, with esophageal varices, normal renal function and free of medications (vasoactive drugs or diuretics) were assigned to 2 different protocols. Protocol 1: 10 patients were randomized to receive octreotide or placebo, as a bolus followed by a continuous infusion. Glomerular filtration rate (GFR) and renal plasma flow (PRF) were measured, in basal conditions and during the drug or placebo administration. Protocol 2: 10 additional patients were randomized in the same way and free water clearance and urinary sodium excretion were again measured in the basal period and during the drug or placebo infusion. Results: After octreotide or placebo administration no significant changes were observed neither in GFR nor in PRF. The free water clearance decreased significantly during octreotide administration $(3.12 \mathrm{ml} / \mathrm{min} \pm 1.04 \mathrm{SE}$ vs $0.88 \pm 0.39, p<03)$. In both protocols no changes in mean arterial pressure were observed. Conclusions: Acute administration of octreotide to cirrhotic patients with portal hypertension, with or without ascites, did not produce any change in glomerular filtration rate or in estimated renal plasma blood flow. However the free water clearance decreased significantly. This effect, under chronic administration, could be clinically important and deserves further studies (Rev Méd Chile 2004; 132: 144-50).

(Key Words: Hypertension, portal; Liver, cirrhosis; Octreotide; Portal pressure)

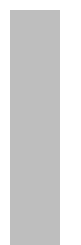

\footnotetext{
Recibido el 23 de abril, 2003. Aceptado en versión corregida el 17 de noviembre, 2003.

Trabajo financiado parcialmente por proyecto Fondecyt, \# 1950-748.

${ }^{1}$ Departamento de Gastroenterología, Clínica Las Condes, Santiago, Chile. ${ }^{2}$ Instituto Chileno-Japonés de Enfermedades Digestivas, Hospital Clínico San Borja-Arriarán, Santiago, Chile. ${ }^{3}$ Unidad de Gastroenterología, Departamento de Medicina, Campus Centro, Facultad de Medicina, Universidad de Chile.

a Tecnóloga Médica
}

Correspondencia a: Dr. Guillermo Silva. Lo Fontecilla 441, Las Condes, Santiago, Chile. Fax: (56-2)-7388062. E mail: mjpc@entelchile.net 
L a administración de octreotido, análogo sintético de la somatostatina, es uno de los tratamientos aceptados en el manejo de la hemorragia por rotura de várices esofágicas ${ }^{1}$. El control del sangrado ocurre a pesar de que el octreotido tiene pocos o ningún efecto sobre la presión portal ${ }^{2} \mathrm{y}$ ha sido atribuido a la inhibición que produce el octreotido de la hiperemia esplácnica postprandial por la liberación de glucagón ${ }^{3}$ y también a la producción de venoconstricción esplácnica, a través de mecanismos no conocidos aún ${ }^{4}$. Las consecuencias de estos efectos sobre la circulación sistémica y renal no son bien conocidas y se han descrito resultados contradictorios en pacientes cirróticos ${ }^{5,6}$.

Se considera que la disfunción renal que puede presentarse en cirróticos, es secundaria a la respuesta homeostática de los sistemas vasoactivos (el eje renina-angiotensina-aldosterona, las catecolaminas y la hormona antidiurética) a la vasodilatación arterial, para mantener la presión arterial dentro de rangos normales. Estos fenómenos ocurren predominantemente en la circulación esplácnica ${ }^{7}$.

La administración de vasoconstrictores arteriales, predominantemente esplácnicos, como la ornipresina y la terlipresina, mejoran la función renal en cirróticos con ascitis e insuficiencia renal. Estos fármacos se han utilizado con cierto éxito, ya sea solos o en combinación con albúmina intravenosa, en el tratamiento del síndrome hepatorrenal tipo 1, a la espera de que se produzca la mejoría espontánea o pueda realizarse el trasplante hepático ${ }^{8}$.

La somatostatina, asimismo, produce vasoconstricción importante en la circulación esplácnica, pero también induce vasoconstricción renal, con depresión de la filtración glomerular, del clearance de agua libre y de la excreción de sodio ${ }^{9}$. Otros autores han encontrado disminución de la excreción renal de agua libre, sin cambios en el clearance de creatinina ${ }^{10,11}$. Puesto que el octreotido es un análogo de la somatostatina, cabe plantearse que pueda producir los mismos efectos sobre el riñón.

En pacientes cirróticos, los efectos de la administración aguda de octreotido sobre el riñón han sido controversiales ${ }^{5,6}$. En un estudio reciente, la administración por 30 días de una forma de octreotido de liberación prolongada no produjo cambios significativos en la función renal ${ }^{12}$. El uso de octreotido, en combinación con agonistas alfa adrenérgicos y expansores de volumen, ha mejorado la hemodinámica sistémica y renal en pacientes con síndrome hepatorrenal tipo 1, aunque la función renal no mejoró totalmente, a pesar de la normalización de los sitemas neurohormonales ${ }^{13}$.

Considerando estos resultados controversiales, el propósito del presente estudio fue el de evaluar, en pacientes con cirrosis, con hipertensión portal, con y sin ascitis, diversos parámetros de función renal, incluyendo el clearance de agua libre, después de la administración aguda de octreotido.

\section{Pacientes y Métodos}

Pacientes: Fueron elegibles para este estudio todos los pacientes cirróticos que consultaron sucesivamente en el Policlínico de Hepatología del Hospital San Borja-Arriarán y que tenían várices esofágicas diagnosticadas endoscópicamente en los 3 meses previos a la consulta y con función renal normal (creatinina $<1,5 \mathrm{mg} / \mathrm{dl}$, examen de orina normal y riñones normales en la ecografía), siempre que cumplieran además con los siguientes criterios: a) abstinencia del alcohol en los últimos 6 meses; b) en caso de existir ascitis, sólo mínima o moderada y sin uso de diuréticos; c) sin antecedentes de hemorragia digestiva alta en el último mes; d) ausencia de trombosis portal o hepatocarcinoma; e) ausencia de valvulopatía o anormalidades contráctiles evaluadas por eco-Doppler; f) ausencia de patología sistémica importante; g) consentimiento informado escrito.

Protocolo de estudio: El estudio incluyó dos protocolos. Protocolo 1: 10 pacientes fueron asignados aleatoriamente para recibir octreotido 0 placebo, en forma doble ciega. En ellos se estudiaron la velocidad de filtración glomerular (VFG) y el flujo plasmático renal (FPR), mediante la medición de clearance de Inulina y para-aminohipurato respectivamente. Protocolo 2: otros 10 pacientes fueron también aleatoriamente asignados para recibir octreotido o placebo y en ellos se midieron la excreción de agua libre, la excreción urinaria de sodio y la excreción fraccional de sodio, tanto en condiciones basales como después de la infusión. 
El motivo de los dos protocolos es que si todas las mediciones se hubieran efectuado en el mismo paciente, el estudio habría demandado un tiempo inaceptablemente prolongado.

Métodos. Al ingreso, a todos los pacientes se les indicó reposo en cama y una dieta que contenía $50 \mathrm{mmol}$ de sodio y $80 \mathrm{mmol}$ de potasio al día. Al segundo día, en el protocolo 1, con el paciente en ayunas, se efectuaron los tests de función renal. Se cateterizaron ambas venas antecubitales, una para la infusión y la otra para obtener muestras. También se instaló una sonda vesical. A continuación se efectuaron los clearances de inulina (IN) y para-aminohipurato (PAH), según Arroyo ${ }^{14}$ y Smith $^{15}$ respectivamente. Se infundió en 10 min un bolo inicial de IN 50 $\mathrm{mg} / \mathrm{kg}$ peso y PAH $8 \mathrm{mg} / \mathrm{kg}$ peso, seguido de una infusión continua de IN $30 \mathrm{mg} / \mathrm{min}$ y PAH 12 $\mathrm{mg} / \mathrm{min}$. Sesenta minutos después de iniciada la infusión se obtuvieron 3 muestras de sangre y orina, cada $30 \mathrm{~min}$. Luego de ello se infundió un bolo de $50 \mathrm{ug}$ de octreotido, o un volumen idéntico de placebo (solución fisiológica) seguido de una infusión continua de $50 \mathrm{ug} / \mathrm{hr}$. Después de una hora se repitieron los clearances de IN y $\mathrm{PAH}$, de acuerdo con metodología ya descrita por nuestro grupo ${ }^{16}$.

En el segundo protocolo, se realizó un clearance de agua libre, administrando una carga acuosa de $10 \mathrm{ml} / \mathrm{kg}$ peso (solución glucosada al $5 \%$ en 45 min. Después de 15 min se midió la diuresis, se descartó esta orina y se administró oralmente un volumen igual al volumen urinario. Luego se obtuvieron 3 muestras de sangre y orina cada $30 \mathrm{~min}$. Todo el procedimiento se repitió después de la administración de octreotido 0 placebo. Las muestras de sangre y orina se conservaron a $-80^{\circ} \mathrm{C}$ hasta que se efectuaran las determinaciones químicas.

Función hepática: se evaluó mediante determinaciones de bilirrubina sérica, albúmina, tiempo de protrombina y las variables clínicas incluidas en el puntaje de Child-Pugh ${ }^{17}$.

Procedimientos analíticos: la IN se midió según el método de Roe et al $^{18}$ y el $\mathrm{PAH}$ según Smith et $\mathrm{al}^{15}$.

Estadística: para calcular las diferencias entre o en los grupos se aplicaron el $t$ test de Student o tests de $\mathrm{t}$ pareados. Los cálculos no paramétricos se hicieron usando los tests de Friedman y Dunn.

\section{Resultados}

1. Datos clínicos generales de los pacientes

a) Protocolo 1: La edad promedio en el grupo total fue $48,3 \pm 11,4$ años, sin diferencias entre los que recibían octreotido o placebo. Siete pacientes fueron hombres, predominando en el grupo octreotido. La etiología de la cirrosis fue alcohólica en 7 casos. El puntaje de Child-Pugh fue 8,2 $\pm 3,1$ en el grupo octreotido y $6,8 \pm 1,8$ en el grupo placebo (NS).

En el grupo octreotido, 3 de los pacientes presentaron ascitis, en comparación con 1 de 5 en

Tabla 1. D atos clínicos generales de los pacientes

\begin{tabular}{|lcccc|}
\hline & $\begin{array}{c}\text { Placebo } 1 \\
(\mathrm{n}=5)\end{array}$ & $\begin{array}{c}\text { Octreotido } \\
(\mathrm{n}=5)\end{array}$ & $\begin{array}{c}\text { Placebo } 2 \\
(\mathrm{n}=5)\end{array}$ & $\begin{array}{c}\text { Octreotido } 2 \\
(\mathrm{n}=5)\end{array}$ \\
\hline Edad (años) & $49,0 \pm 14,9$ & $47,6 \pm 8,2$ & $51,0 \pm 16,6$ & $62,0 \pm 7,3$ \\
Sexo (Hombre/Mujer) & $2 / 3$ & $5 / 0$ & $4 / 1$ & $3 / 2$ \\
Alcoholismo & $1 / 5$ & $5 / 5$ & $3 / 5$ & $0 / 5$ \\
Ascitis & $1 / 5$ & $3 / 5$ & $2 / 5$ & $0 / 5$ \\
PAM (mmHg) & $84 \pm 23$ & $85 \pm 14$ & $82 \pm 17$ & $84 \pm 20$ \\
Creatinina (mg/dl) & $1,0 \pm 0,2$ & $0,9 \pm 0,2$ & $1,1 \pm 0,3$ & $0,9 \pm 0,1$ \\
Child-Pugh score & $6,8 \pm 1,8$ & $8,2 \pm 3,1$ & $7,2 \pm 1,9$ & $7,2 \pm 1,6$ \\
\hline
\end{tabular}

PAM=presión arterial media 
el grupo placebo. La presión arterial media basal fue casi idéntica en ambos grupos ( $85 \pm 14$ vs $84 \pm 23 \mathrm{mmHg}$ ). La creatinina promedio fue $1,1 \pm 0,3$ $\mathrm{mg} / \mathrm{dl}$ en el grupo total.

b) Protocolo 2: la edad promedio en el grupo total fue $56,2 \pm 13,2$ años, sin diferencias entre octreotido y placebo. Siete pacientes eran hombres, con distribución similar en ambos grupos. Hubo 3 alcohólicos, todos en el grupo placebo. El puntaje de Child-Pugh fue similar en ambos grupos $(7,2 \pm 1,9$ y $7,2 \pm 1,6)$. Los niveles de creatinina en el grupo total fueron de 0,9 $\pm 0,1 \mathrm{mg} / \mathrm{dl}$.

2. Efectos del octreotido sobre la filtración glomerular y el flujo plasmático renal (Protocolo 1)

Los clearances basales de IN y PAH no mostraron diferencias significativas entre ambos grupos (Tabla 1). Después de la administración de octreotido o placebo no se observaron cambios significativos en los clearances de IN ni PAH (Figura 1, A y B). Tampoco se modificó la presión arterial media.

3. Efectos del octreotido en el clearance de agua libre y en la excreción de sodio (Protocolo 2)

El clearance de agua libre disminuyó significativamente después de la administración de octreotido (Figura 2), de 3,12 $\pm 1,04$ (ES) $\mathrm{ml} / \mathrm{min}$ a $0,88 \pm 0,39$ ( $p<, 03)$, pero no cambió en el grupo placebo. La excreción fraccional de sodio aumentó después de la infusión de octreotido, pero sin alcanzar significación estadística (octreotido basal $2,5 \pm 4,2 \%$ vs postinfusión $4,8 \pm 7,4$ ).

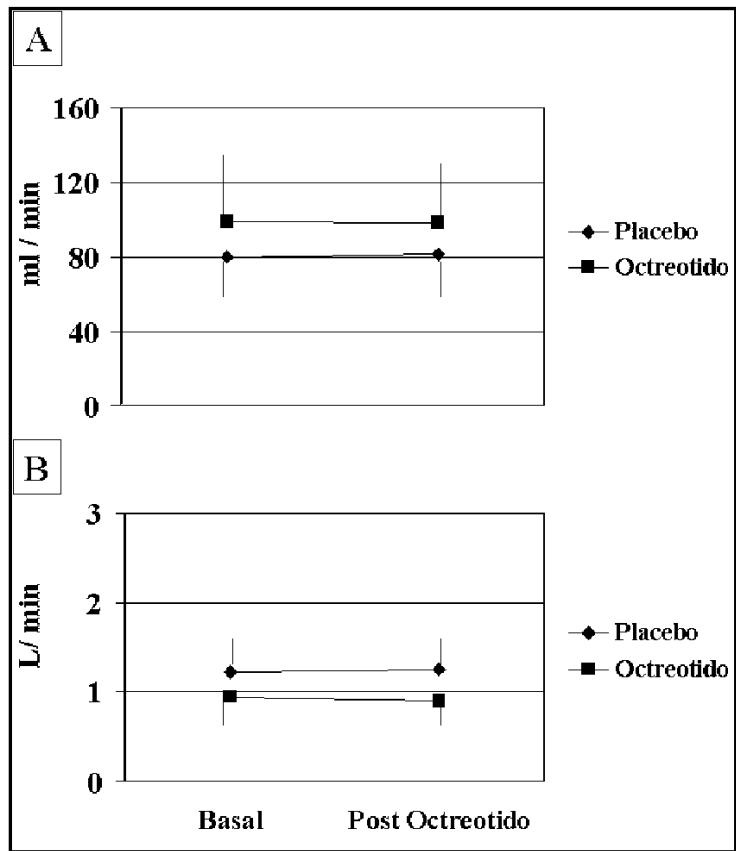

Figura 1. Efectos del octreotido y placebo sobre la velocidad de filtración glomerular y flujo plasmático renal. A. Variaciones en la velocidad de filtración glomerular (clearance de Inulina) antes y después de la administración de octreotido o placebo. Promedio \pm desviación estándar (en líneas verticales). Los cambios no fueron significativos. B. Variaciones en el flujo plasmático renal (clearance de para-aminohipurato), en las mismas condiciones. Promedio \pm desviación estándar (en líneas verticales). No se observaron diferencias significativas.

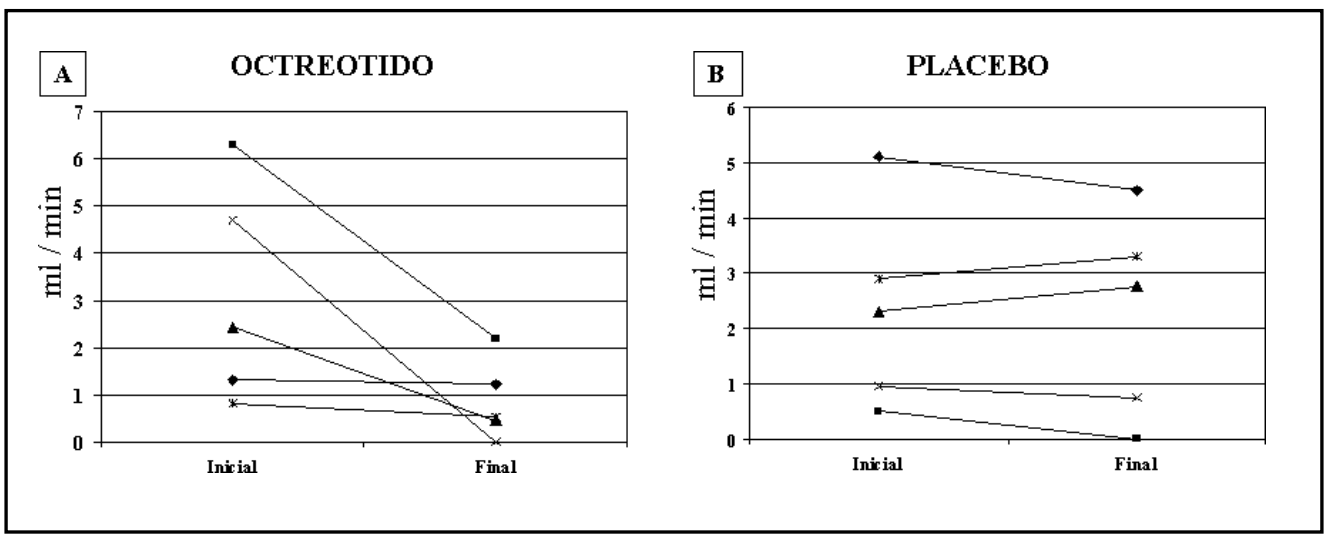

FiguRA 2. Excreción de agua libre después de la administración de octreotido o placebo. Variaciones individuales en el clearance de agua libre. Las líneas unen los clearances antes y después del octreotido (A) 0 placebo (B), en el mismo paciente. Se observa una clara disminución del clearance en el grupo octreotido (para valores ver texto). 


\section{DisCUSIÓN}

Los resultados principales de este estudio muestran que el octreotido, administrado como bolo y seguido de una infusión continua, no produjo variaciones significativas en la VFG ni en el FPR, pero sí produjo una importante y significativa disminución del clearance de agua libre. Estos cambios no parecen deberse a variaciones en la hemodinámica sistémica, ya que la presión arterial media no se modificó.

El uso del octreotido en el tratamiento del sangrado variceal en cirróticos se basa en los efectos conocidos de la somatostatina en la hemodinámica esplácnica, donde reduce el flujo sanguíneo portal y colateral y disminuye la presión portal mediante una selectiva vasoconstricción arteriolar esplácnica ${ }^{19,20}$. A diferencia de la somatostatina, el octreotido ha mostrado efectos variables sobre la hemodinámica esplácnica, específicamente sobre la presión portal, el flujo sanguíneo por la vena azygos y la presión variceal21-23. Recientemente se ha descrito que después de la administración de un bolo, se produce una disminución marcada, pero transitoria, de la presión portal y del flujo portal colateral, junto con un aumento de la presión arterial media, lo que sugiere un efecto directo vasoconstrictor sistémico. Sin embargo, la adición de una infusión continua no mantiene ni prolonga estos efectos.

En modelos experimentales de cirrosis e hipertensión portal, el octreotido mejora la perfusión renal y aumenta la excreción de sodio, lo que sugiere un efecto inhibitorio sobre la secreción de renina-aldosterona, así como normalización de la arginina-vasopresina ${ }^{24-26}$. La administración de octreotido, combinada con midodrina - un agonista alfa adrenérgico- y con expansión de volumen, produjo una mejoría significativa de la hemodinámica sistémica y renal y un aumento en la

\section{REFERENCIAS}

1. Garcia-Tsao G. Current management of the complications of cirrhosis and portal hypertension: variceal hemorrhage, ascites and spontaneous bacterial peritonitis. Gastroenterology 2001; 120 : 726-48. excreción urinaria de sodio en una pequeña serie clínica ${ }^{13}$.

El glucagón aumenta la VFG y el FPR tanto en humanos como en modelos experimentales ${ }^{27,28}$ y es probablemente un mediador importante de la vasodilatación renal que ocurre en algunas condiciones asociadas con niveles plasmáticos elevados de glucagón, como la diabetes mellitus insulinodependiente ${ }^{29,30}$. La cirrosis hepática es otra condición asociada con niveles plasmáticos elevados de glucagón ${ }^{31}$. Podría, por lo tanto, especularse que el octreotido, que produce un efecto inhibitorio sobre los niveles plasmáticos de glucagón, pudiera empeorar la función renal a través de este mecanismo.

Según nuestros resultados, el octreotido no modificó la VFG ni el FPR, lo que sugiere que no se produjo redistribución del flujo sanguíneo desde el territorio esplácnico al renal. Este resultado fue independiente del grado de insuficiencia hepática, ya que 3 de los 5 pacientes estudiados tenían ascitis y se comportaron de la misma manera que los que no la presentaban.

Por otra parte, el clearance de agua libre disminuyó significativamente. Este resultado no concuerda con otros datos experimentales ${ }^{32}$, pero una disminución similar ha sido observada también durante la administración de somatostatina ${ }^{9}$, lo que podría sugerir un probable efecto agonista de la $\mathrm{ADH}$ en el túbulo renal. Este efecto podría ser más deletéreo en pacientes con sangrado variceal activo y función renal anormal.

En resumen, la administración aguda de octreotido a pacientes cirróticos, con o sin ascitis, no produjo cambios ni en la velocidad de filtración glomerular ni en el flujo plasmático renal, pero la excreción de agua libre disminuyó significativamente. Este efecto, en condiciones de administración más prolongada, podría ser clínicamente importante y merece ser estudiado.

2. Escorsell A, Bandi JC, Andreu V, Moitinho E, Garcia-Pagan JC, Bosch J et al. Desensitization to the effects of intravenous octreotide in cirrhotic patients with portal hypertension. Gastroenterology 2001; 120: 161-9.

3. Albilos A, Rossi I, Iborra J, Liedo JL, CaLieja JL, BARRIOs C ET AL. Octreotide prevents postprandial 
splanchnic hyperaemia in patients with portal hypertension. J Hepatol 1994; 21: 88-94.

4. Hadengue A. Somatostatin or octreotide in acute variceal bleeding. Digestion 1999; 60 (Suppl 2): 31-41.

5. Mountokalakis T, KaLivretakis N, MayopoulouSymvouldou D, Karvountzis G, Tous G. Enhancement of renal function by a long-acting somatostatin analogue in patients with decompensated cirrhosis. Nephrol Dial Transplant 1988; 3: 604-7.

6. Sabat M, Guarner C, Soriano G, Bulbena O, Novela MT, Ortiz J Et aL. Effect of subcutaneous administration of octreotide on endogenous vasoactive systems and renal function in cirrhotic patients with ascites. Dig Dis Sciences 1998; 43: 2184-9.

7. Schierer RW, Arroyo V, Bernardi M, Epstein M, HenRIKSEN JH, Rodës J. Peripheral arterial vasodilation hypothesis: a proposal for the initiation of renal sodium and water retention in cirrhosis. Hepatology 1998; 8: 1151-7.

8. Wong F, BLENDis L New challenge of hepatorenal syndrome: prevention and treatment. Hepatology 2001; 34: 1242-51.

9. Gines A, Salmeron JM, Gines P, Jiménez W, Saló J, PIERA C ET AL. Effects of somatostatin on renal function on cirrhosis. Gastroenterology 1992; 103: 1868-74.

10. Berthold H, Del Pozo E. Antidiuretic effect of Sandostatin (SMS 201-995) in healthy volunteers. Acta Endocrinol 1989; 120: 708-14.

11. Walker BJ, Nelstrop JA, Joekes AM. Somatostatin and water excretion. Lancet 1983; 1: 1101-2.

12. Ottesen LH, Aagaard NK, Kiszka-Kanowitz M, Rehung M, Henriksen JH, Pedersen EB et al. Effect of a long-acting formulation of octreotide on renal function and renal sodium handling in cirrhotic patients with portal hypertension: A randomized, double-blind, controlled trial. Hepatology 2001; 34: 471-7.

13. Angeli P, Volpin R, Gerunda G, Craighero R, Roner P, Merenda R ET AL. Reversal of type 1 hepatorenal syndrome with the administration of midodrine and octreotide. Hepatology 1999; 29: 1690-7.

14. Arroyo V, Rodes J, Gutiérrez-Lizarraga MA, ReverT L. Prognostic value of spontaneous hyponatremia in cirrhosis with ascites. Dig Dis 1976; 21: 249-56.

15. Smith HW, Finkelstein N, Aliminosa L, CRawford B, GRABER M. The renal clearences of substituted hippuric acid derivatives and other aromatic acids in dog and men. J Clin Invest 1945; 24: 388-404.

16. Silva G, Segovia R, Ponce R, Backhouse C, Palma M, RoBLERO JP ET AL. Effects of 5-isosorbide mononitrate and propranolol on subclinical hepatic encephalopathy and renal function in patients with liver cirrosis. Hepato-Gastroenterology 2002; 49: 1357-62.

17. Pugh RNH, Murray-Lyon IM, Dawson JL, Pietroni MC, Wiwams R. Transection of the oesophagus for bleeding oesophageal varices. Br J Surg 1973; 60: 646-9.

18. Roe JH, Epstein JH, Goldstein NP. A photometric method for the determination of inulin in plasma and urine. J Biol Chem 1949; 178: 839-45.

19. Bosch J, Kravetz D, Mastai R, Navasa M, Silva G, CHESTA J ET AL. Effects of somatostatin in patients with portal hypertension. Hormone Res 1988; 29: 99-102.

20. Bosch J, Kravetz D, Rodes J. Effects of somatostatin on hepatic and systemic hemodynamics in patients with cirrhosis of the liver: comparison with vasopressin. Gastroenterology 1981; 80: 518-25.

21. ERIKSSON LS, BRundin T, SüDERLund C, WAHzen J. Haemodynamic effect of a long acting somatostatin analogue in patients with liver cirrhosis. Scand J Gastroenterol 1987; 22: 919-25.

22. Lin HC, Tsai YT, Lee FY, Lee SD, Hsia HC, Lin WJ ET AL. Hemodynamic evaluation of octreotide in patients with hepatitis B - related cirrhosis. Gastroenterology 1992; 103: 229-34.

23. Moler S, Brinch K, Henriksen JH, Becker U. Effect of octreotide on systemic, central and splanchnic haemodynamics in cirrhosis. J Hepatol 1997; 26: 1026-33.

24. Jonassen TE, Christensen S, Sprensen AM, MarcusSen N, Fly rberg A, ANDReasen F et al. Effects of chronic octreotide treatment on renal changes during cirrhosis in rats. Hepatology 1999; 29: 1387-95.

25. Albillos A, Colombato LA, Lee Fy, Grossman RJ. Octreotide ameliorates vasodilatation and $\mathrm{Na}$ retention in portal hypertensive rats. Gastroenterology 1993; 104: 575-9.

26. Wang SS, Lee FY, Wu SL, Hwu CM, Chien CH, Lee SD ET AL. Effects of long term administration of octreotide on sodium retention and atrial natriuretic peptide in carbon tetrachloride induced cirrhotic rats. J Hepatol 1997; 26: 1128-34. 
27. JohanNSEN J, Lie M, KiIL F. Effect of glycine and glucagon on glomerular filtration and renal metabolic rates. Am J Phisiol 1997; 233: F61-F66.

28. CASTEuno P, HuRT W, De Fronzo RA. Regulation of renal hemodynamics by plasma aminoacid and hormone concentrations. Kidney In 1987; 32: 515-20.

29. Parving HH, Christiansen JS, Noer I, Tronier B, Mogensen CE. The effect of glucagon infusion on kidney function in short term insulin dependent juvenile diabetics. Diabetologia 1980; 350-4.

30. Pedersen MM, Christiansen SE, Christiansen JS, Pedersen EEB, Megensen OE, Orskor H. Acute effects of a somatostatin analogue on kidney function in type 1 diabetic patients. Diabetic Med 1990; 7: 304-9.

31. Silva G, Gomis R, Bosch J, Casamitjana R, Mastai R, NAvaSA M ET AL. Hyperglucagonism and glucagon resistance in cirrhosis. Paradoxical effect of propranolol on plasma glucagon. J Hepatol 1988; 6: 325-31.

32. Mountokalakis T, Levy M. Effect of a selective octapeptide analogue of somatostatin on renal water excretion in the dog. Metabolism 1985; 34: 408-9. 